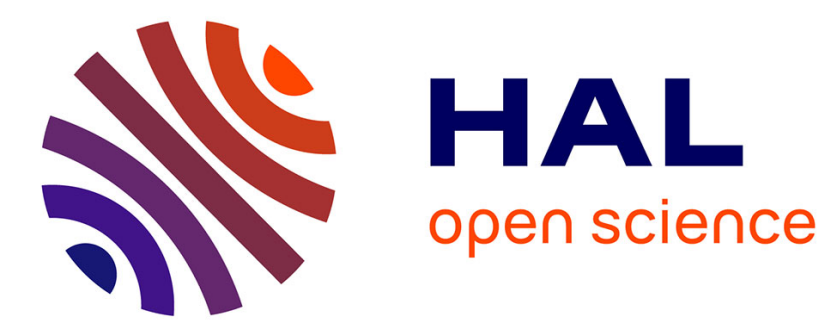

\title{
Documents sur l'oppidum de Jastres-Nord, à Lussas (Ardèche) \\ Yves Burnand
}

\section{To cite this version:}

Yves Burnand. Documents sur l'oppidum de Jastres-Nord, à Lussas (Ardèche). Gallia - Fouilles et monuments archéologiques en France métropolitaine, 1977, 35 (2), pp.271-278. 10.3406/galia.1977.1567 . hal-01939350

\section{HAL Id: hal-01939350 https://hal.science/hal-01939350}

Submitted on 25 Feb 2020

HAL is a multi-disciplinary open access archive for the deposit and dissemination of scientific research documents, whether they are published or not. The documents may come from teaching and research institutions in France or abroad, or from public or private research centers.
L'archive ouverte pluridisciplinaire HAL, est destinée au dépôt et à la diffusion de documents scientifiques de niveau recherche, publiés ou non, émanant des établissements d'enseignement et de recherche français ou étrangers, des laboratoires publics ou privés.

\section{(이) $\$$}

Distributed under a Creative Commons Attribution - NonCommercial - NoDerivatives| 4.0 


\title{
DOCUMENTS SUR L'OPPIDUM DE JASTRES-NORD A LUSSAS (Ardèche)
}

\author{
par Yves BURNAND
}

Le site de Jastres-Nord, appelé la Grande Muraille, est situé à l'ouest du territoire communal de Lussas (arrondissement de Privas, département de l'Ardèche) (fig. 1) 1 . $^{1}$ Il se trouve dans la partie septentrionale du plateau calcaire jurassique des Gras, vaste table karstique qui s'étend entre les larges vallées de l'Ardèche à l'ouest et de son affluent l'Auzon a l'est'. Celte portion septentrionale du plateau des Gras, limitée au nord et au sud par deux profonds ravins creusés par des ruisseaux affluents de l'Ardeche, est couramment appelée plateau de Jastres et elle renferme les deux sites de Jastres-. Yord au nordouest et de Jastres-Sud au sud-ouest.

Jastres-Yord occupe un promontoire triangulaire situé à l'extrémité nord-ouest du plateau de Jastres; ce promontoire, à la pointe dirigée vers le nord comme le souligne particu-

1 A. Brave, Carte archéologique de la Gaule romaine, fasc. $\mathrm{XV}$, Ardeche, p. $62, \S 2$, a fait firmere a tort ce site sous le $n^{\circ} 59$ de son répertoire, attribue a la commune d'Aubenas. Non seulement le plateau de Jastres n'apparlient pas à celle-ci, mais il n'en est même pas limitrophe : comme le montre le plan cadastral (voir l'extrait donne ici, infra, fig. 2, il esl limiti al l'o. par les communes de saint-lerivat ef de saint-l)idirr-sous-Aubenas, la commune d'Aubenas sarrêtant avant le cours de l'Ardiche. Ie sito de Jastres-sud fait partie du territoire communal de Iavillediru.

2 Frederic Romax, Le Bas-linarais, 1950, p. 9-10, avec fig. 2, p. 7 . lierement bien le plan cadastral de la commune de Lussas, s'avance entre la vallée de l'Ardèche a l'ouest, la vallée du ruisseau de Louyr au nord et une digitation de celle-ci a l'est, tandis qu'au sud il se rattache au plateau par un léger ensellement; l'altitude en est de peu supérieure a $310 \mathrm{~m}$ et ne dépasse $320 \mathrm{~m}$ en aucun point. C'est un site classique d'éperon barré : pour les deux longs côtés et la pointe du triangle les défenses naturelles suffisent, car la pente est trìs forte a l'ouest, où le plateau domine la vallée de l'Ardèche par une falaise abrupte de $130 \mathrm{~m}$ de dénivellation, très raide encore au nord (pas de l'Échelette), moins marquée mais encore suffisante à l'est; en revanche. aucune défense naturelle n'existe au sud et la base du promontoire a été barrée par un rempart. du sud-ouest au nord-est, qui fait partie du paysage traditionnel du plateau au point d'avoir fait appeler ce site la Grande Muraille par les gens de la contrée. Ce rempart est cependant demeuré longtemps assez mal connu en dehors de sa silhouette caractéristique. Mais depuis un siècle les auteurs régionaux s'y sont intéressés et son existence a été rappelée par une série de documents graphiques, dont la qualité s'est améliorée en fonction des progries que la connaissance du site faisait avec le temps. Il a paru intéressant de présenter la progression de cette connaissance d'une construction régionale 


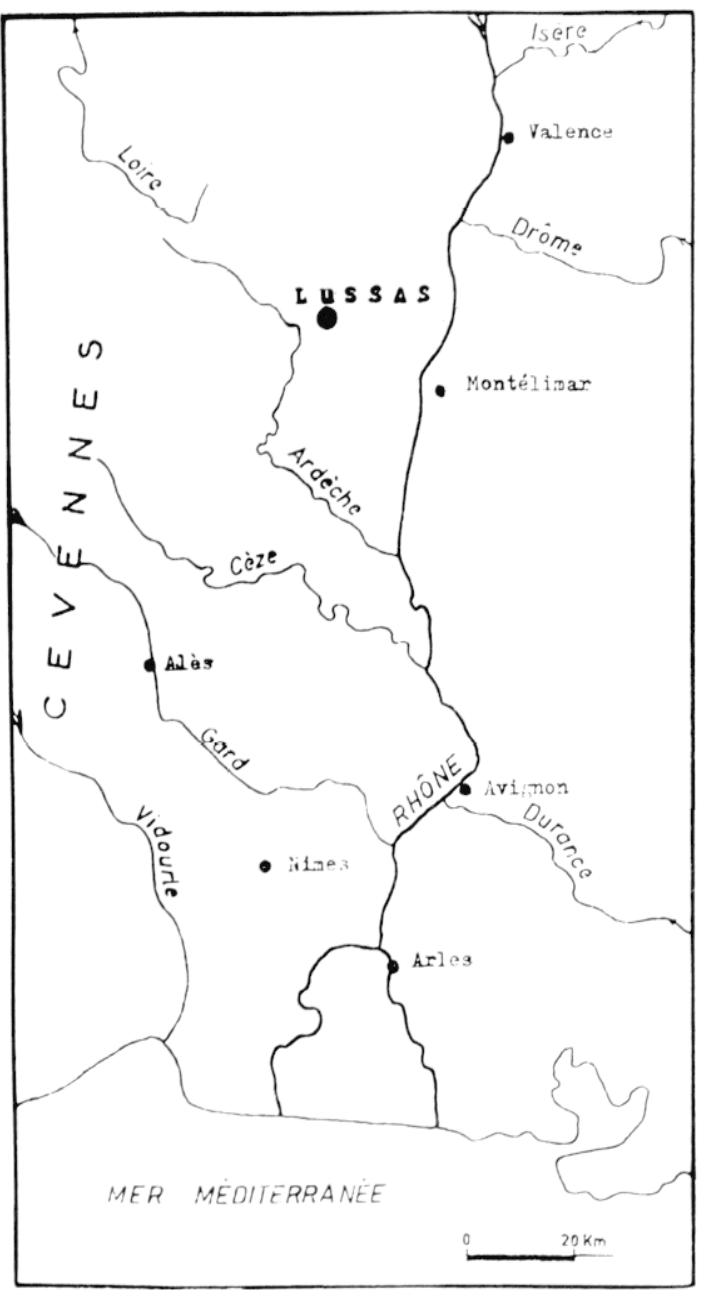

I Siluation de la communo de Lussas.

remontant à la plus haute Intiquite, connaissance demeurée extérieure jusqu'aux sondages et au levé qui ont été exécutés tout récemment. a notre initialive.

Le nom de Jastres est apparu dans les chartes des xive et $x^{-e}$ siecles sous la double dénomination de Rancus de Jastrias et de Ciradus de Jastrias et il a paru tentant de le meltre en rapport, en raison du caractiere d'oppidum du lieu, avec le lalin castra, ramp ${ }^{3}$.

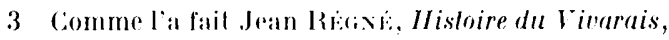
I, Largentiere, 1914, p. 240, n. 3 inote additionnelle all texle du chanoine J. Rouchier!. Coest le chanoine Rouchier ibidem, p. 23x, n. 2., qui a donme les denx formes anciemues du nom de Jastres, sans en fournir de réferences précises. Il n'existre pas rncore de dictionnaire
Le site de l'éperon de Jastres-Yord a été signalé pour la première fois, de facon incidente, dans la liltérature régionale par le rhanoine Rouchier en $18622^{4}$. I)ans le dernier quart du xixe siecle l'érudit, polycraphe vivarois Albin Mazon, qui publiait généralement sous le pseudonyme de docteur Francus, lui a consacré un passage de l'un de ses récits de randonnées a travers le département, en mème temps qu'au site voisin de Jastres-sud : il a rapporté alors pour Jastres-Nord le nom local de "los Tourres". "les Tours " el a pensé effectivement pouvoir reconnaitre sur le rempart "la trace de trois tours rondes et d'une carrée $n^{5}$.

la premiere représentation graphique du rempart de Jastres-Nord est celle qu'a donné le plan cadastral de la commune de Lussas ${ }^{6}$. Entre la falaise à pic qui surplombe a l'est la vallée de l'Ardeche, servant de limile communale entre Lussas d'une part et siant-Privat et siant-Didier-sous-Aubenas d'autre part, et le rhemin de l'Échelette Vieille, une limite cadastrale oblique, de direction très différente des autres. matérialise dans le parcellaire rural la survivance de l'ancien rempart en lant que limile. Blle en evoque ainsi la forme générale, du sud-ouest au nord-est, a l'exclusion de tout détail (tigr. 2).

In peu moins schématique apparait la figuration du rempart sur la carte an $1 / 2$ ). (0)(0) de l'Institut géographique national (fig. :3). Il y est représenté dislinctement sous la forme d'une levée de lerrain : ce serait une erreur sur sa nature que de l'interpréler comme une levée de terre sa struclure étant toute diflérente: mais rela rend bien l'aspect de large Lalus recouvert par une végétation huissonnante qu'il présentail jusqu'aux travaux que nous $y$ avons conduits.

topographique pour le departement de l'Ardeche ; celuici posside loutefois un fichier des moms de lieux aux Archives departementales a Privas, mais il n'y figure aucune reference, précise pour Jastres frenseignement aimablement communique par ll me le conservateur des services d'archives de l'Ardeche.

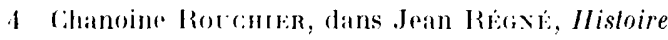
du Vinarais, I, p. 23x.

5 b) Fraxios 'Allin Mazox, Voyage le long de la riviere d Ardeche, Privas, 16xi), p. 33.

6 Plan cadastral do la commune de lussas, section I, dite du Gras, fuartier de l'Echeledte. 


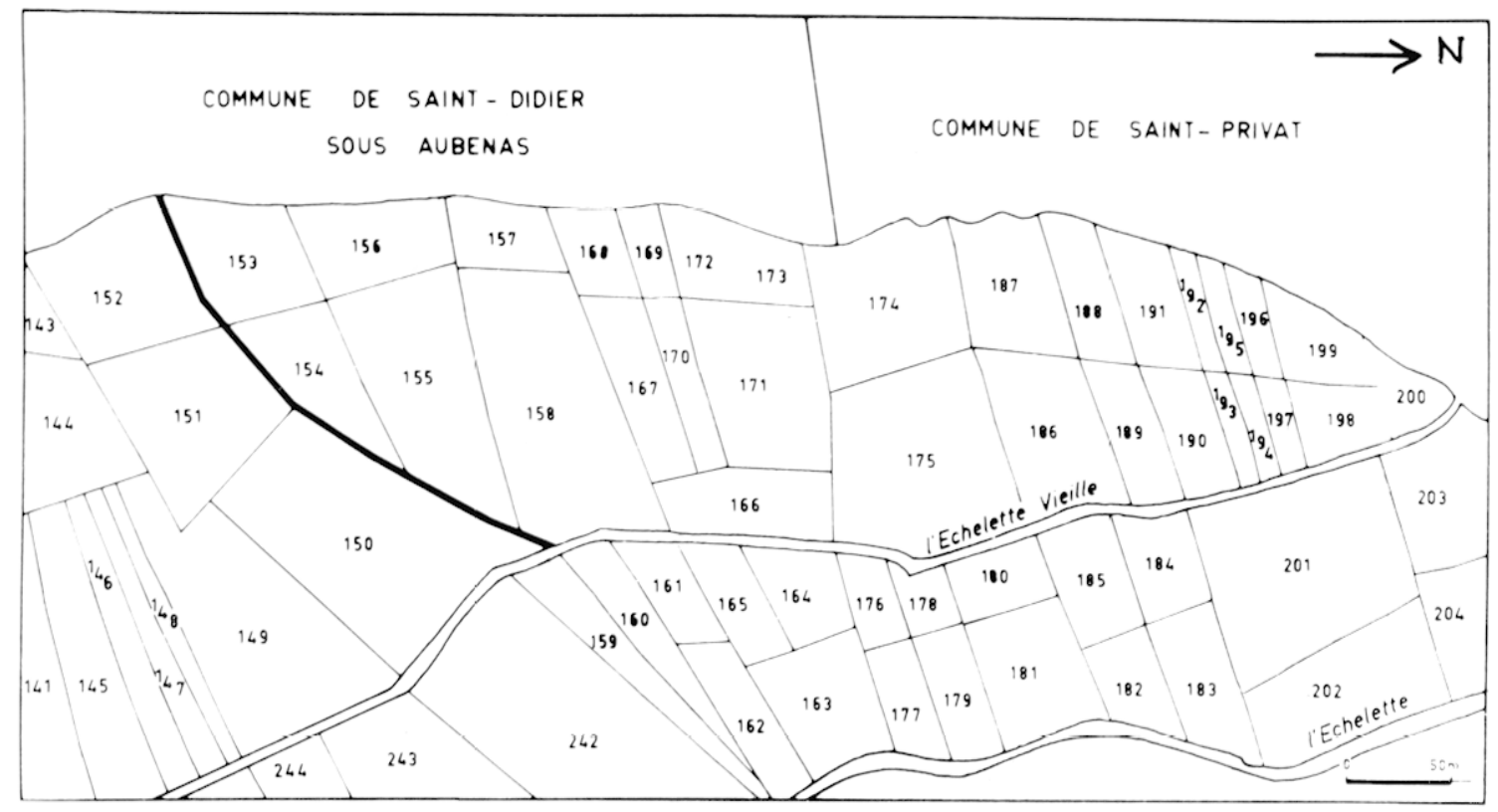

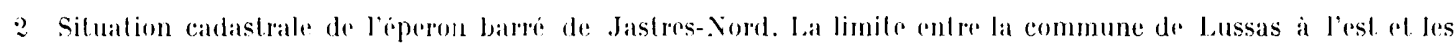

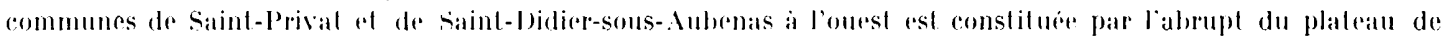
Jastres sur la vallé de l'Ardeche. la pente orientale du plateau, beancoup moins raide, est constilue par l'espace

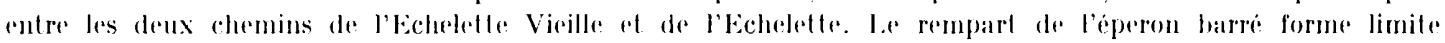

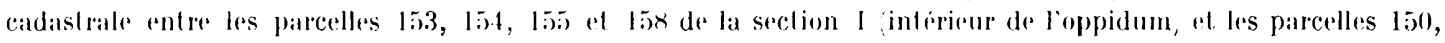
151 et 152 á l'exterieur de l'oppidum ; le lait indiquant la limile a ate epaissi, mais la direction de cette limite est parfaitement aherante par rapport anx autres lignes cadastrales de cette section et materialise netfement sur Ir plan la présence du rempart sur le terrain.

\section{Illustration non autorisée à la diffusion}

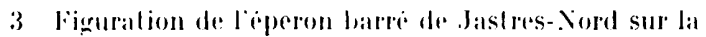
rarte de leance atl les.ome de l'Institul giographique national carte XXIX-3x, Aubenats, $\mathrm{n}^{\prime \prime} 1-2$, .
En revanche, les photographies aériennes presentent plus exactement un long ruban de couleur claire caractéristique d'un pierrier. révélant ainsi la nature véritable du rempart. ()n peut y distinquer certains détails : interruption du rempart a l'ouest avant la falaise qui surplombe l'Ardeche (donc possibilité d'une porte), rentlement du pierrier par places (done existence probable de bastions ou de tours). Ces délails apparaissent sur les clichés de la photothéque de l'Institut géographique national ${ }^{7}$ comme sur ceux qui ont été pris par l'aviation alliée au cours de la seconde querre mondiale, en préparation au débarquement des troupes alliées sur le littoral méditerranéen en 1944, et qui sont conservés dans la photothéque de l'École Française de

7 Phototherfue de l'Institul geographique national. Couverture acrienne de la France, .11 ission $69 \mathrm{FR} 1790$ 100, cliche n" 1556. 

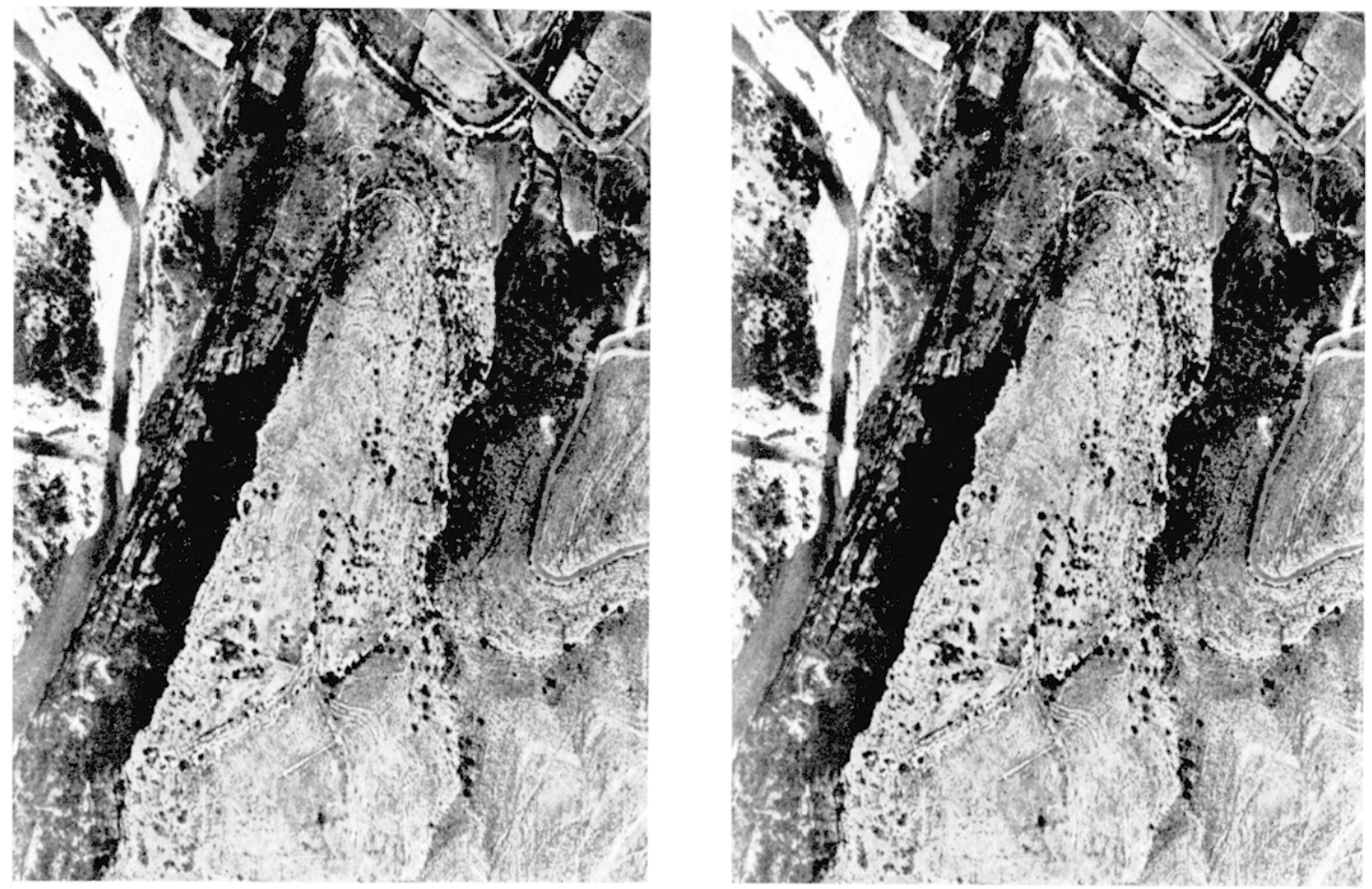

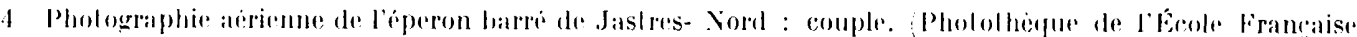

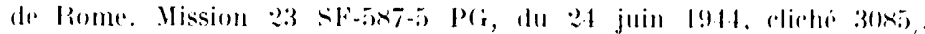

Rome ${ }^{8}$; ils sont rependant plus nets sur ces derniers, en raison de la maigreur de la végétation a l'époque des prises de vues (fig. 4 et 5 ).

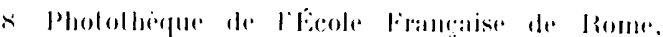

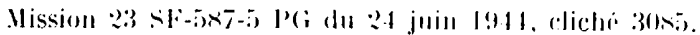
Celte sonrer de docenmentation. venanl des missions arrienmes accomplies en 19.1.1 par les avialions milibaires anglaise at amerieaine all-dessus des régions meritlie. nales et cernl rales de la france, a ser rendue ulilisable par Ie tratail dîlentificalion of de classement dì an regrette colonel Jean Barader. Celui-ci en al signali l'interêt dans la prósentation yulil m a faile à dells reprises, sons les litres: Alu service de larchéslogie gallo-romaine, les pholographies ariennes de latialion interallise dipusses a l Eicole Francaise de Rome, dans: Melanges de likcole firuncaise de kome, ix, Igtifi, p. $267-27: 2$; Les photographies ariennes prises en France par l'ariation interalliée, dípossies a l'Ecole Francaise de Rome, dans diallia, 24, 19666, 1. 5.29-531. Nous avons pu consuller ces cliches an cours diun séjour d'etudes a l'Ecole franeate de lome en novernbere 1973 a t mous lenons a exprimer notre grali-

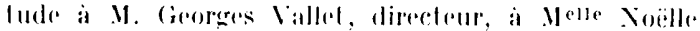

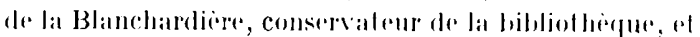

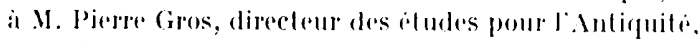

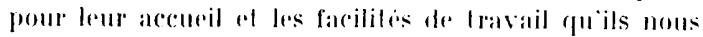
ont accorderes.

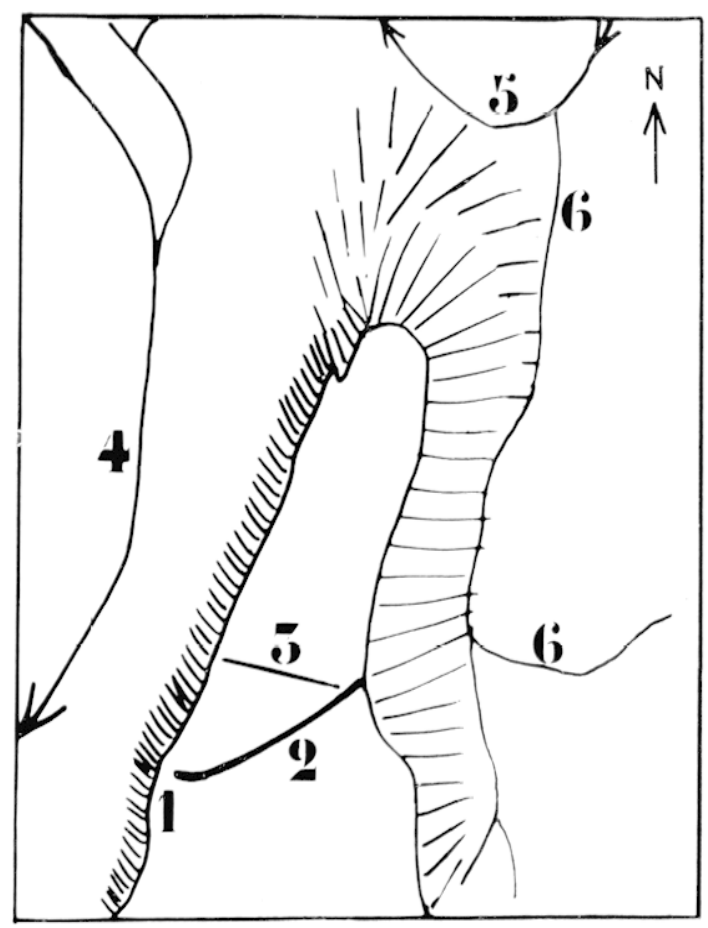

¿) Caldgue d'interpretation de la photographie acrienne: 1 porte de loppidum?, 2 rempart, 3 mur transtersal, 4 Ardeche, इ) ruissean de lomer, 6 ruisseall du vallom de l'Feheletle. 
Ciest diapres la photographie abienne de l'Institut geographique national que le rommandant Raoul Pérol a donné un roquis du rempart dans son manuserit sur l'arrhéologie du département de l'Ardiches. Dépourva dorientation et d'orhelle, re roquis figure quatre bastions el une tour ronde a l'extrémile orientale du remparl. L'auleur y a ausis reporte un mur lransiversal. bien visible sur les pholographes aérennes, mais qui ne semble pas avoir appartenu au svisteme defensif anlique : en oulre. il a situe la jonction de re mur avec le rempart an niveau de la derniere lour a l'est. alors qu'il n'existe pas de racoord entre les deux et que la direction rectiligne suivie par ce mur transicersal rencontre le rempart sensiblement plus a l'ouest; entin. il a gratifie ce mème mur de deux bastions sur la face sud el d'un donjon a son extremite nort-ouest. alors que rien n'autorise a restituer res lrois alements ${ }^{10}$. Il ne sagil que d'un maluais requis d'interpretation de pholographie arerienne (fig. (i).

En plus de ce plan róduit el relevant davanlage de limagination que de la réalite, le mème érudit a fourni une coupe st ratigraphique de l'intérieur de la derniere tour a l'est. Elle a éte due aux observalions faites en 1962 par M. Gérard Barras el le regrette Jean-Claude Doux. sous la diredion de M. Henri sianmadell : roupe depourve deechelle, mais l'indicalion de lat hauteur des couches a ete portée directement pour charune en ordonnee (lig. 7 ).

Le premier plan serieux du rempart a che donné en 1969 par 1. II. . I. Hoog dans un rapide lorilige d'oppidums du territoire francais $^{12}$ : plan oriente, mais pourva d'une

9 II sagit do trois ahiers manuscrits . Ms . A. Ms

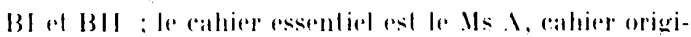

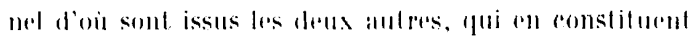
des mises all poinl parlielles : il comple 300 pages

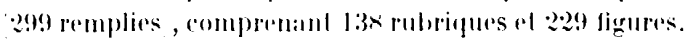
Coms an avons domene ane presenlation ol les grandes lignes doune ilude critifure du materiel documenlatere ainsi massemble : Yors Broxaxn, Les "manuscrils

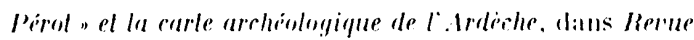
du Virarais, 1976, :2. 1). (i)

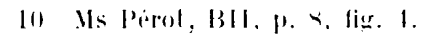

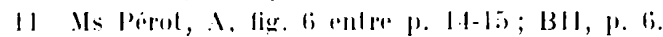

1: A. H. A. Hooni, A trample of French Ilill Forls,

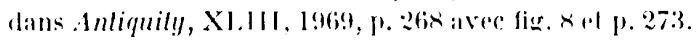

ichelle seulement approximative fomme la indiqué d'ailleurs l'auteur. il romporte la figuration de six bastions ou lours. I a masse du pierrier constitué par le rempart est représentee par une surface poinlillée d'égale largeur sur loule sa longueur, alors quäil n'en est rien au sol : de plus. dans celte masise l'autecir a represente en deux traits continus parallies les courtines du rempart. domnant ainsi limpression d'un rempart simple a double parement. alors qu'en fail la structure du rempart est loule dilfirente el beauroup plus romplexe. En revanche, le mur transversal. dont nous avons déja note qu’il étail tres probablement elranger an systeme défensif antique. ne figure pas sur le plan de A. H. .1. Honge ce qui montre que ret auteur partageail deja ce sentiment (fig. $\gamma_{\text {. }}$. Dans une notice consacre au plateau de Jastres dans l'Mnliquile .I. Menri saumade a noté l'existence de sepl lours, en les voyant loutes rondes: matis il n'a fourni aucun plan du rempartis.

le "repertoire des momuments el découvertes a dressé par Il. André Blanc dans le fascicule départemental de la carte archéologique de la Gaule romaine consarere a l'Ardiche fait mention du rempart de Jastres-Xord, mais en incluant par erreur ce site dans la nolice consacrée a la rommune d'Aubenast4. Bien que postérieur de six ans a l'article de . I. I. Hooge le travail de M. Blanc ignore rel article dans la hibliographie du sile éréduile a 1 . Mazon comme dans la bibliographie densemble en tibe du volume. Aussi l'information sur la ronstitution du rempart en reste-t-elle a celle que l'on pouvait avoir sur lui en 1885, puisque, a la suite de 1. Mazon, II. A. Blanc continue à lui allribuer seulement "Irois lours rondes et une carrée". (In ne roit pas, de plus. comment les murs antiques. qui barrent en oblique le promontoire comme

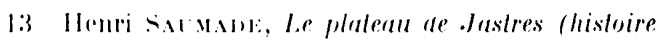
ol prihisloire), dans Rerue de lo tor. des tinfants el . Imis

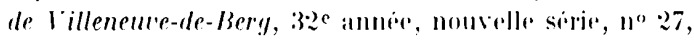
1972 , p. 3.4-35, Jasl res- Nimed.

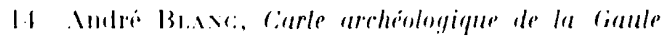

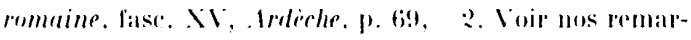
ques faites ici sufra. p. :70, n. I localisalion communalle reromion. 


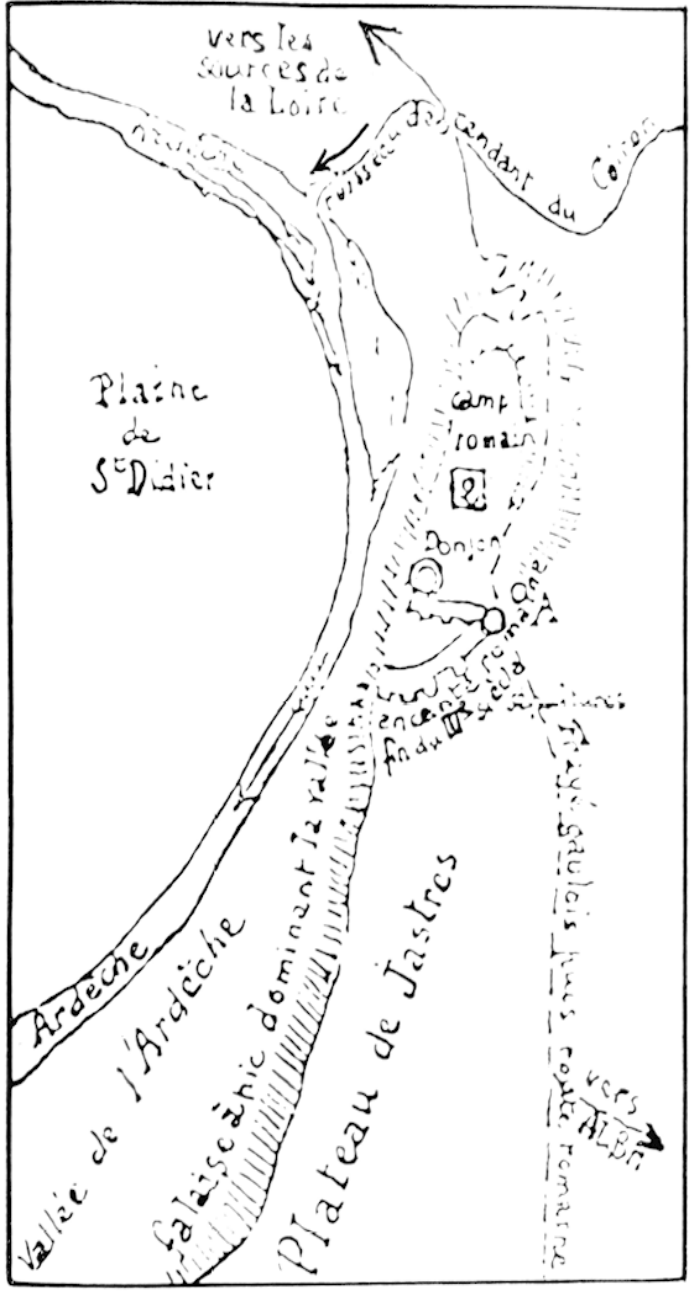

6 Cirounis de l'eperon barre de Jastres- Vord dans le manuscrit Perot Be ip. x, fig. 1 .

le montrent le plan cadastral (fig. 2). la carte de France au 1/25.000) (fig. 3), la photographie aérienne (fig. 4) et le roquis de l'article de A. I1. A. Hoog (fig. 8), peuvent former « un rectangle de $100 \mathrm{~m}$ sur $200 \mathrm{~m}$ ) (?).

los recherches sur le tracé du rempart sont a l'origine du plus récent relevé de cellui-ri et elles ont permis une exacte représentation de l'aspect acluel du site de Jastres-Nord et des éléments connus du systime défensif. Iors de la reconnaissance méthodique que nous avions conduite sur le sile le 2 septembre 197:3. un plan sommaire du systime défensif avait, été établi par MY. Alain I)upuy, dessinateur industriel a I yon, et Guy Iauswald, géomitre a Xancy, dans le seul but de servir de document

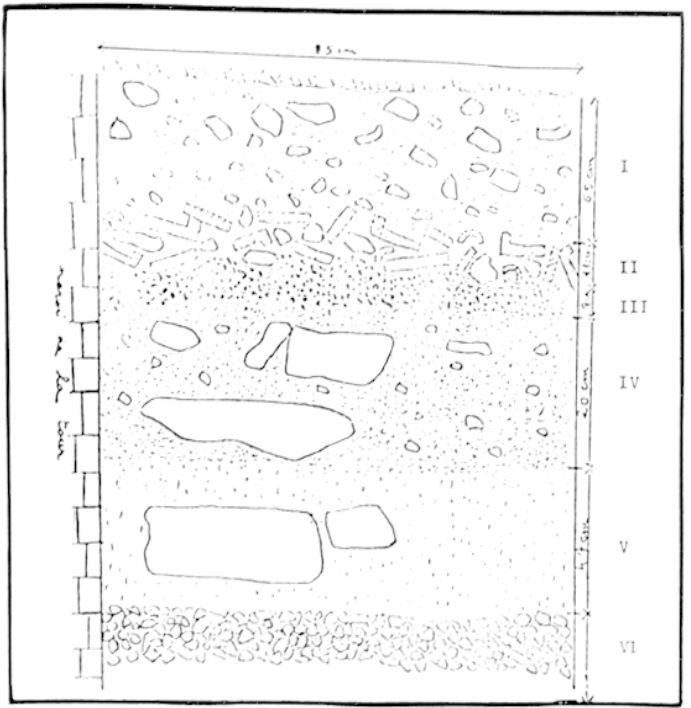

7 Straligraphie du remplissage de la tour VIl dans Ir manuscrit Perot 13: p. 6, eblablie par H. Sammale

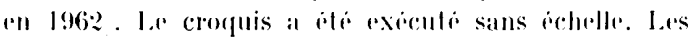
numeros en chiffres romatins portis à droile correspomdent anx mumbros figurant an mème amplacerment dans les manuscrils Perot. la legende, inscrite igalement a droile dams ress mamuserils, a ble reportion ci-dissous :

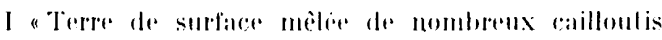
anguleux"

II " Fragments de fegulan of d'imbrices"

III " couche de chathous dincendie at de cendres"

IV "louche formen de fragments de biton ordinato" a de berlon a luilean"

$V$ a Terre argileuse foncin mêler dans sa seconde moitio ef vers sil base do fraements de poterie dousage combant. Cortains sont de la lencente. Guelques lessons de dolimm, ossements de pores et dovins debris de cussint",

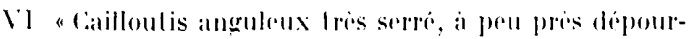
vil de lerre. Couche sterile"

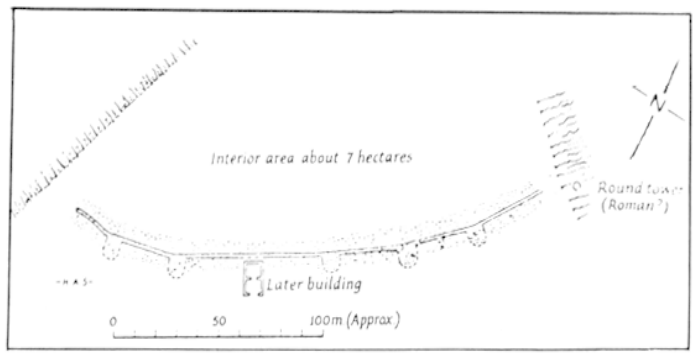

$x$ Le Lace du remparl de Jastres- Vord dams laarlicle dr. 1. II. 1. Hoon it timple of French Hill-Jorts, dans Intiquily. XI.JII, 1969, p. 268, fig. * . 


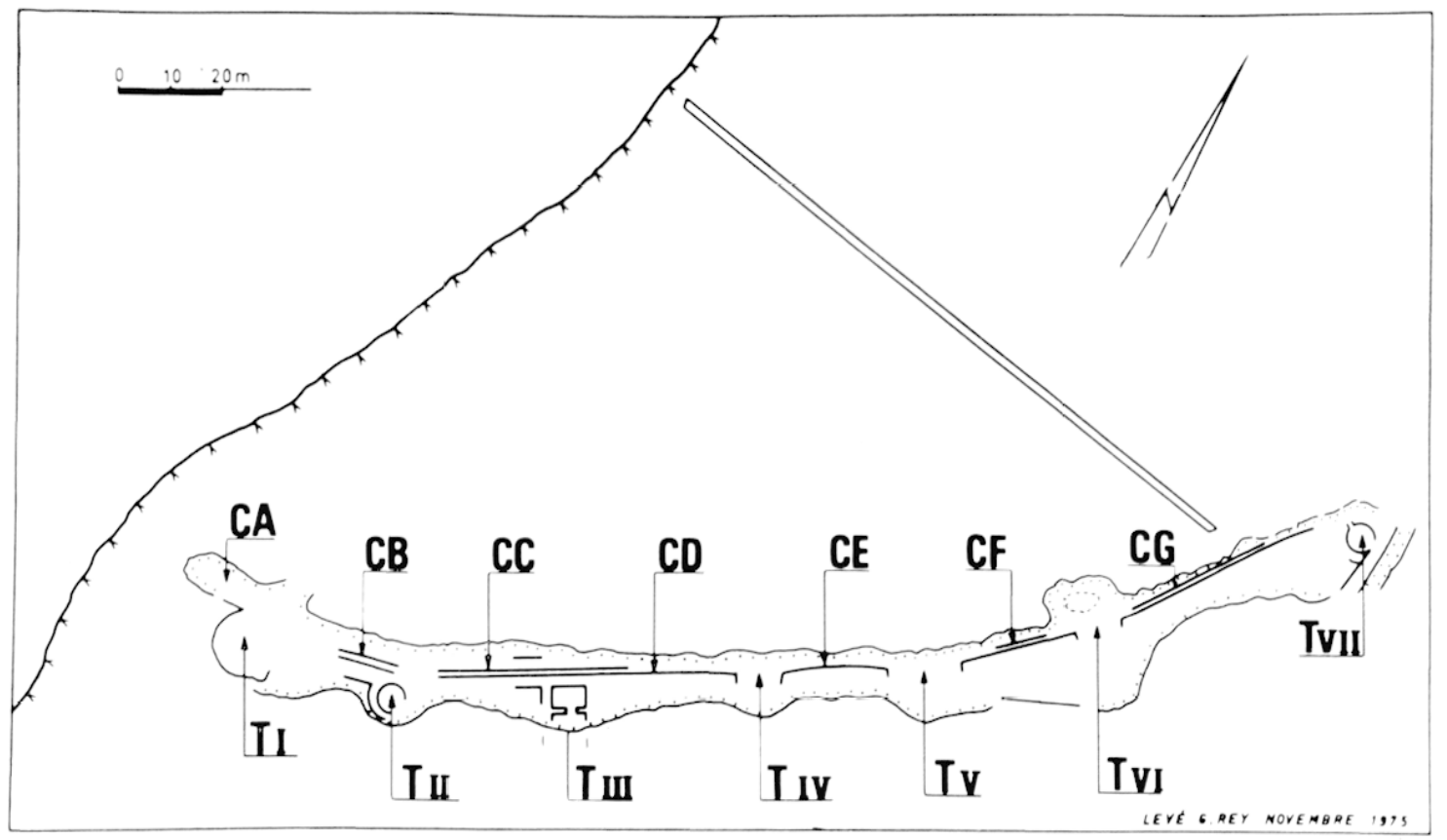

9 Plan du rempart de loppidum de Jastme- Norel leve apres les travaux de lg7t par M. Gaston Regi.

de travail pour une rampanne ullerieure. Cone campagne de sondages a dé entreprise en divers points du tracé du rempart en août $1974^{15}$; a l'issue de celle-ri, des relevés de détail furent fails par M.V. Bernard Manipoud et bominique Tavernier; mais aurun levé d'ensemble du rempart n'avait alors pu atre effectur.

C'est celle lacune que nous avons entendu combler en 1975) gràce a l'aide du département de l'Arrieche el a la bonne volonlé de $\mathrm{V}$. Gaston Rey, directeur adjoint honoraire du servire du Cardastre du département de l'Ardeche (fig. 9). I.e levé du rempart de l'éperon barré de Jastres-Cord a été exéculé origrinellement. au 1 /one ; on y a fait figurer le mur transversal non antique seulement i titre de repire. I.es conlours du pierrier qui sólive sur l'empla-

li cis recherches furenl enlreprises du 19 all 31 aont lo7t par une equipe du croupe universilater nanceien d'Antiquites nationales, aree lassistance de

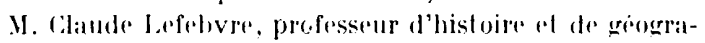
phie an lycere Robert schuman a Nort ef grace an concours financier de l'l niversile de Nancy II et de la Ville de Sancy : Sorge I Axcre, Informations archoologiques. Circonscription de Rhône-dlpes, dans Ciallia, 33, 1975, p. 533-531, fig. 10. cement du rempart onl éte cernes par un trait. continu léger. L'irrégularité de ces rontours laisse deviner, par la succession des parties moins larges ef des épaississements, l'alternance des courlines et des bastions ou tours écroulés vers l'extérieur, a la faveur de la pente. A l'intérieur du pierrier seules ont été représentées les parties des parements qui daient visibles sur les courtines el sur les hastions ou lours avant les travaux d'aoùt 1974 ou qui l'ont été rendues par ceux-ci : parement non pas toujours simplement double, romme A. II. A. Hoog l'avait fait figurer sur son plan de 1969. mais plus souvent triple comme l'ont prouvé divers sondagres faits en 1974. L'indication des différents déments du rempart a éte portée sur chacun d'eux au moyen de la codification tris simple adoptée dis aont 1974 el progressant d'ouest en est de 1 a i pour les sept éléments de rourtines. de I a VII pour les sept hastions ou tours; car d'une parl ces eléments de flanquement ont été netlement individualisés, la jonction de rhacun aver les courlines ayant été reconnue sans équivoque : d'autre part il $y$ a bien sept bastions ou lours, et non six comme lavait cru el représenté . I. II. 1. Hoog 
en 1969 : la tour numérotée III sur notre plan avait été considérée par cet auteur comme une construction postérieure au rempart et totalement indépendante de celui-ci, alors que les travaux de 1974 ont permis de constater que les murs des còtés occidental et oriental de cette construction ne sont pas accolés au parement extérieur de la courtine, mais engagés dans celle-ci.

Les travaux qui seront poursuivis ultérieurement sur le tracé du rempart bénéficient donc désormais d'un plan de base exact, sur lequel pourront être reportées les découvertes au fur et à mesure que progressera la connaissance de ce système défensif. Il paraît tout à fait souhaitable que l'on puisse disposer d'un dossier bibliographique el figuré complet de celte sorte pour chacun des oppida de celte récrion, qui en est riche : res dossiers permettraient de constater le progris des connaissances en ce domaine, inégal selon les sites: leur comparaison aiderail à l'établissement d'une typologie extérieure de ces systèmes défensifs, datant le plus souvent de l'époque de l'indépendance des peuples gaulois, mais en plus d'un cas remaniés, comme a Jastres-Nord, à l'époque gallo-romaine.

Yves Berixind. 